\title{
Applied Voltage Dependence of Carbon Radical in Electric Double Layer Capacitor Measured by in situ ESR Spectroscopy and AC Cyclic Voltammetry
}

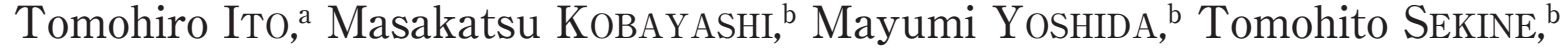 \\ Kazuhiro TachiBAnA, ${ }^{\mathrm{a}, *}$ Tatsuo Nishina, ${ }^{\mathrm{a}}$ and Tateaki OGata ${ }^{\mathrm{a}}$ \\ ${ }^{\mathrm{a}}$ Graduate School of Science and Engineering, Yamagata University (4-3-16 Johnan, Yonezawa-shi, Yamagata 992- \\ 8510, Japan) \\ ${ }^{b}$ Faculty of Engineering, Yamagata University (4-3-16 Johnan, Yonezawa-shi, Yamagata 992-8510, Japan)
}

Received November 29, 2009 ; Accepted January 12, 2010

\begin{abstract}
In this study, the behavior of carbon radicals has been observed in a model cell of an electric double layer capacitor (EDLC) by using an in situ electron spin resonance (ESR) method and ac cyclic voltammetry (CV). The maximum ESR signal intensity of the carbon radical was observed during the anodic sweep of the applied voltage using the simultaneous measurement of the ESR, the impedance and the CV. It was found that the degradation progress of the EDLC was mainly due to the change in the concentration of the carbon radical, and that its phenomenon was not observed by $\mathrm{CV}$.
\end{abstract}

Key Words : Electric Double Layer Capacitor (EDLC), Ionic Liquid, Electron Spin Resonance (ESR), Carbon Radical

\section{Introduction}

Various approaches are being performed for the achieving a low-carbon society. ${ }^{1)}$ A "smart grid" to integrate information with energy is expected, because the expansion in the use of renewable energy should be attempted. ${ }^{1)}$ An electric double layer capacitor (EDLC) has been actively researched in order to meet the needs of energy storage devices that require rapid charging and discharging, especially for the electric power resurrection use. ${ }^{2-4)}$ Therefore, it is important to investigate the deterioration mechanism of the EDLC, and to evaluate its cycle-life. The electrolyte solution of the electrochemical devices decomposes during the charging process. ${ }^{2,5)}$ In order to clarify the mechanism of this decomposition, it has been analyzed by in situ or ex situ Fourier Transform Infrared spectroscopy (FTIR). ${ }^{5-10)}$ However, it is difficult to analyze the carbon by FTIR, because its absorbance is high. In general, it is known that many radicals exist in the surface of the activated carbon used as an EDLC electrode, and it is assumed that the change in the concentration of the carbon radical may be related to the oxidation of the electrolyte solution, the electrode material and itself by the electrical charge and discharge. ${ }^{11)}$ If one assumes that the internal resistance is increased by oxidizing the solvent, the influence of the solvent can be evaluated using the ionic liquid as the electrolyte solution not containing the solvent. Ionic liquids have been widely investigated as non-flammable and non-volatile electrolytes for electrochemical devices, as such advantages have been also beneficial for EDLCs as the energy storage devices. ${ }^{2,3,5,12,13)}$ In this study, the behavior of the carbon radical has been observed in a model EDLC cell using an in situ electron spin resonance (ESR) method and ac cyclic voltammetry. The purpose of this study is to investigate the relationship between the internal resistance of the EDLC and the generation of the radical by oxidation of the electrolyte solution.

\section{1 Electrolyte solution}

\section{Experimental}

A neat ionic liquid of 1-(methoxymethyl)-1-methylpyrrolidinium tetrafluoroborate $\left(\mathrm{MMP}^{\left.-\mathrm{BF}_{4}\right)}\right.$ at a concentration of $5.7 \mathrm{M}\left(\mathrm{M}=\mathrm{mol} \cdot \mathrm{dm}^{-3}\right)$, a propylene carbonate $(\mathrm{PC})$ solution of MMP-BF $\mathrm{M}_{4}$ at a concentration of $1.5 \mathrm{M}$ and a PC solution of $N, N, N$-triethyl- $N$-methylammonium tetrafluoroborate (TEMA-BF ${ }_{4}$ ) at a concentration of $1.4 \mathrm{M}$ were used as the electrolyte solutions.

\subsection{The EDLC model cell for the ESR}

The EDLC model cell for the ESR was made as follows. Two electrodes were placed in the cylindrical sample tube (i.d. $3.5 \mathrm{~mm}$ ) and used as shown in Fig. 1. The carbon-coated aluminum sheet was used as the electrode; the nickel wire ( $0.5 \mathrm{~mm}$ outer diameter) was used as the lead wire. The carbon-coated aluminum sheet had a 50 $\mathrm{mm}$ length, $3 \mathrm{~mm}$ width, and $0.1 \mathrm{~mm}$ thickness. The activated carbon prepared from the hydrocarbon oil was used as the carbon. The carbon was mixed with a polytetrafluoroethylene binder, and it was painted on both sides of the aluminum sheet with the dimensions of a 30 $\mathrm{mm}$ length and the $3 \mathrm{~mm}$ width. This sheet was connected to the lead wire by spot welding and used as the electrode. A thin sheet of cellulose of extremely high purity was used as the separator. The separator and two electrodes were placed in the cylindrical sample tube. The working electrode and counter electrode were placed at a distance of 1.5 and $11.5 \mathrm{~mm}$ from the bottom of the cylindrical sample tube, respectively. This cylindrical 


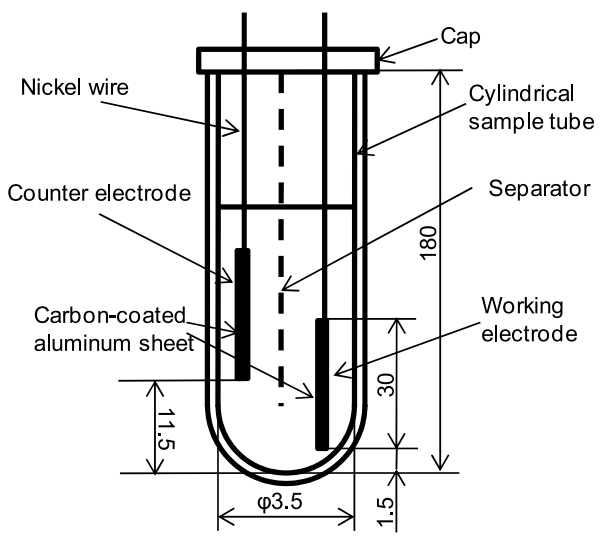

Fig. 1 Schematic of the EDLC model cell.

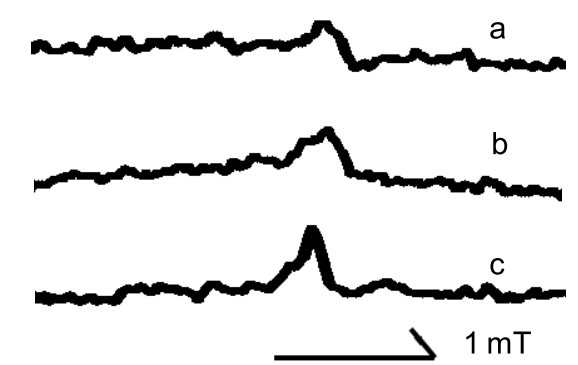

Fig. 2 ESR Spectra of EDLC model cell containing $5.7 \mathrm{M}$ $\mathrm{MMP}-B F_{4}$ (a), $1.5 \mathrm{M}$ MMP-BF $4 / \mathrm{PC}$ (b) and $1.4 \mathrm{M}$ TEMA$\mathrm{BF}_{4} / \mathrm{PC}$ (c).

sample tube containing $0.8 \mathrm{~mL}$ of the various electrolyte solutions was sealed using a cap.

\section{3 AC cyclic voltammetry system combined with in} situ ESR

This system consisted of the EDLC model cell, the ac cyclic voltammetry circuit, the ESR spectrometer (RE3X, JEOL, Japan) and the data logger (GL900, Graphtec, Japan). The ac cyclic voltammetry used a function generator (1946A, NF Corp., Japan), potentiostat (HA-211, Hokuto Denko Corp., Japan), adder circuit (home-built) and lock-in amplifier (LI5640, NF Corp., Japan). The voltage $E_{\mathrm{dc}}$ and the current $I_{\mathrm{dc}}$ of the potentiostat were recorded through the low-pass filter (3611, NF Corp., Japan; filter frequency, $0.5 \mathrm{~Hz}$ ) for the cyclic voltammetry (CV). The EDLC model cell was inserted at the distance of $3 \mathrm{~mm}$ from the upper part in the resonant cavity. The magnetic field $B$ of the ESR was fixed indicating the value of the peak of the ESR signal intensity $S$. The voltage $E_{\mathrm{dc}}$, the current $I_{\mathrm{dc}}$, the conductance $X$, the susceptance $Y$, and the ESR signal intensity $S$ were recorded by the data logger. The resistance $R$, the capacitance $C$ and the impedance $Z$ were calculated from the conductance $X$ and the susceptance $Y$. The relative ESR signal intensity $r$ was calculated from the quotient of the ESR signal intensity $S$ for each time and its intensity $S$ at 0 seconds. The ac cyclic voltammetry conditions were as follows: scan cycle time, $180 \mathrm{~min}$; frequency of excitation signal, $79 \mathrm{~Hz}$; amplitude of excitation signal, $100 \mathrm{mV}$ p-p; time constant in lock-in amplifier, $0.3 \mathrm{sec}$; and the applied voltage $E_{\mathrm{dc}}$ was cycled between -2.5 and $2.5 \mathrm{~V}$.

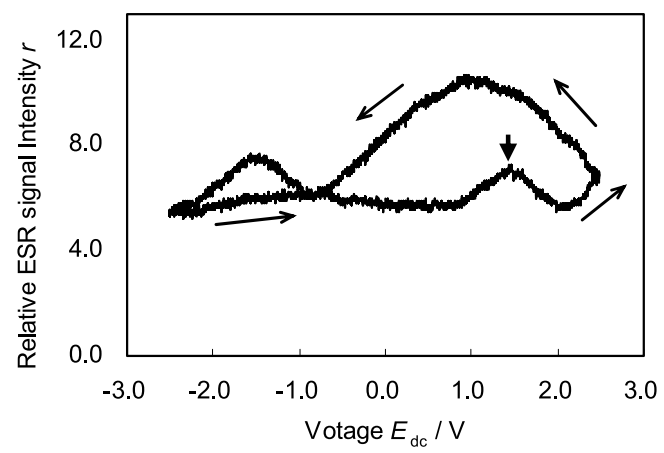

Fig. 3 Relative ESR signal intensity $r$ plotted versus the applied voltage $E_{\mathrm{dc}}$ for the EDLC model cell containing the electrolyte solution of $5.7 \mathrm{M} \mathrm{MMP-BF}{ }_{4}$.

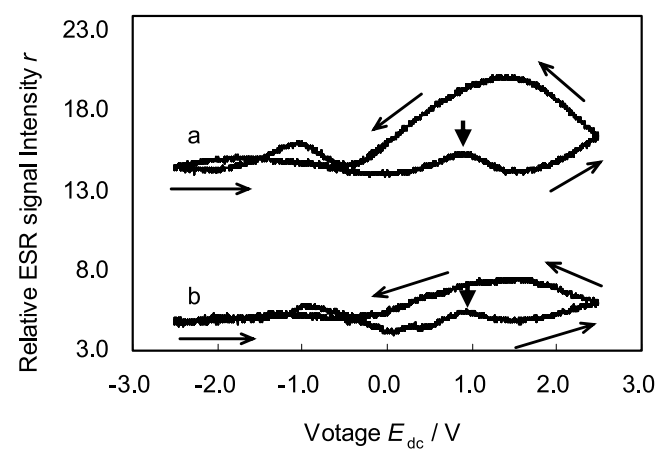

Fig. 4 Relative ESR signal intensity $r$ plotted versus the applied voltage $E_{\mathrm{dc}}$ for the EDLC model cell containing the electrolyte solution of $1.5 \mathrm{M} \mathrm{MMP}-\mathrm{BF}_{4} / \mathrm{PC}$ (a) and $1.4 \mathrm{M}$ $\mathrm{TEMA}^{-\mathrm{BF}_{4}} / \mathrm{PC}$ (b).

\section{Results and Discussion}

3. 1 Evaluation of EDLC model cell for ESR measurement

It is first necessary to confirm that the EDLC model cell was able to be measured by ESR, because the model cell contained an electrolyte solution with a high dielectric loss. This problem was solved by adjusting the insertion distance in the resonant cavity to $3 \mathrm{~mm}$. The ESR conditions were as follows: RF power, $4 \mathrm{~mW}$ at $9.1654 \mathrm{GHz} ; B_{0}$, $327.0 \mathrm{mT}$; time constant in field sweep, $0.1 \mathrm{~s}$; magnetic field sweep speed, $20 \mathrm{mT} / \mathrm{min}$; magnetic field sweep width, $3 \mathrm{mT}$; magnetic field modulation width, $0.25 \mathrm{mT}$ at $100 \mathrm{kHz}$. Figures 2a, 2b and 2c show the ESR spectra obtained from the EDLC model cell containing the $5.7 \mathrm{M}$ ${\mathrm{MMP}-\mathrm{BF}_{4}, 1.5 \mathrm{M} \mathrm{MMP}_{-} \mathrm{BF}_{4} / \mathrm{PC} \text { and } 1.4 \mathrm{M} \text { TEMA-BF }}_{4} / \mathrm{PC}$ solutions, respectively. The $\mathrm{g}$ value of 2.0028 was calculated from each ESR spectra, indicating that these signal peaks were identified in the radical of the carbon center. This finding indicated that the EDLC model cell could be used for the in situ ESR measurement.

3. 2 Simultaneous measurement of the AC cyclic voltammogram using the in situ ESR spectra

Figure 3 shows the relationship between the relative ESR signal intensity $r$ and the applied voltage $E_{\mathrm{dc}}$ of the

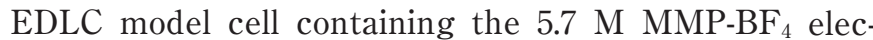
trolyte solution. Figures $4 \mathrm{a}$ and $4 \mathrm{~b}$ show the relationships between the relative ESR signal intensity $r$ and the applied voltage $E_{\mathrm{dc}}$ of the EDLC model cell containing the 1.5 M MMP-BF $/ \mathrm{PC}$ and $1.4 \mathrm{M}$ TEMA-BF $/ \mathrm{PC}$, respec- 
Table 1 Resistance $R$, Capacitance $C$ and Impedance $Z$ at Cycle Number of EDLC Model Cell Containing Various Electrolyte Solutions.

\begin{tabular}{|c|c|c|c|c|}
\hline Electrolyte solutions & Cycle number & $R, \Omega$ & $C, \mu \mathrm{F}$ & $Z, \Omega$ \\
\hline \multirow[t]{3}{*}{$5.7 \mathrm{M} \mathrm{MMP}^{-\mathrm{BF}_{4}}$} & 1 & 89.7 & 18.2 & 142.8 \\
\hline & 2 & 88.5 & 18.6 & 139.9 \\
\hline & 3 & 86.8 & 18.8 & 137.7 \\
\hline \multirow[t]{3}{*}{ 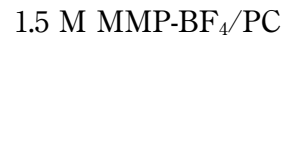 } & 1 & 33.4 & 32.3 & 70.7 \\
\hline & 2 & 34.2 & 30.8 & 73.8 \\
\hline & 3 & 34.9 & 29.8 & 76.1 \\
\hline \multirow[t]{3}{*}{$1.4 \mathrm{M}$ TEMA-BF $4 / \mathrm{PC}$} & 1 & 73.2 & 30.1 & 99.2 \\
\hline & 2 & 74.3 & 29.9 & 100.3 \\
\hline & 3 & 75.4 & 29.5 & 101.8 \\
\hline
\end{tabular}

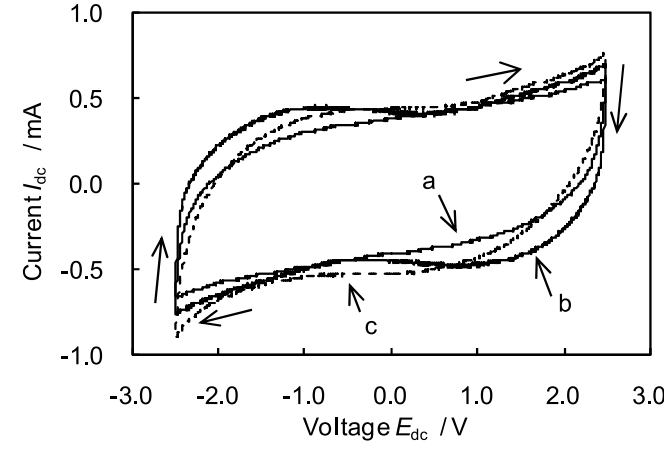

Fig. 5 Cyclic voltammogram of EDLC model cell for electrolyte solution containing 5.7 M MMP-BF 4 (a), $1.5 \mathrm{M}$ $\mathrm{MMP}_{-\mathrm{BF}} / \mathrm{PC}$ (b) and $1.4 \mathrm{M}$ TEMA-BF ${ }_{4} / \mathrm{PC}(\mathrm{c})$.

tively. The relative ESR signal intensity $r$ of the $5.7 \mathrm{M}$ $\mathrm{MMP}-\mathrm{BF}_{4}$ electrolyte solution has a maximum value at a $1.5 \mathrm{~V}$ applied voltage $E_{\mathrm{dc}}$ during the anodic sweep. The relative ESR signal intensities $r$ of the $1.5 \mathrm{M}$ MMP$\mathrm{BF}_{4} / \mathrm{PC}$ and $1.4 \mathrm{M}$ TEMA-BF $/ \mathrm{PC}$ electrolyte solutions have same maximum value at a $0.9 \mathrm{~V}$ applied voltage $E_{\mathrm{dc}}$ during the anodic sweep. The peak current $I_{\mathrm{dc}}$ of the $\mathrm{CV}$ could not be observed for the voltage at the maximum of the relative ESR signal intensity $r$ as shown in Fig. 5. Table 1 shows the $\mathrm{CV}$ cycle number, the resistance $R$, the capacitance $C$ and the impedance $Z$ of the EDLC model cell containing the various electrolyte solutions. The resistance $R$ of the $5.7 \mathrm{M} \mathrm{MMP-BF}$ decreased as the $\mathrm{CV}$ cycle number increased. However, the resistance $R$ of the samples containing PC increased as the CV cycle number increased. These findings indicate that the maximum voltage of the relative ESR signal intensity $r$ of the electrolyte solution containing $\mathrm{PC}$ was lower than the electrolyte solution without the $\mathrm{PC}$, and that its results were not observed by CV. It is suggested that the degradation progress of the EDLC is mainly due to the resistance $R$ increased with the generation of the carbon radical of the electrode by oxidation of the solvent.

\section{Conclusion}

The EDLC model cell could be used by the in situ ESR method. The in situ ESR spectroscopic analyses of the EDLC electrochemical oxidation were carried out. The relative ESR signal intensity $r$ of the electrolyte solution of the neat ionic liquid has a maximum value at a $1.5 \mathrm{~V}$ applied voltage $E_{\mathrm{dc}}$ during the anodic sweep, but the relative ESR signal intensity $r$ of the electrolyte solution of the ionic liquid containing a PC solution has a maximum value at $0.9 \mathrm{~V}$. The applied voltage dependence of the reaction was able to be found by using the simultaneous measurement of the ESR, the impedance and the CV, although the dependence has not been observed using the usual $\mathrm{CV}$ method. The resistance $R$ of the neat ionic liquid decreased as the $\mathrm{CV}$ cycle number increased, but the resistance $R$ of the ionic liquids containing PC increased. These findings indicated that the internal resistance increased with the generation of the carbon radical of the electrode by oxidation of the solvent.

\section{Acknowledgement}

We thank Otsuka Chemical Co., Ltd., for providing of the electrolyte solutions and the electrode materials.

\section{References}

1) H. Tomihara, SURTECH Directory, p.29 (2009).

2) T. Sato, G. Masuda, and K. Takagi, Electrochim. Acta, 49, 3603 (2004).

3) K. Yuyama, G. Masuda, H. Yoshida, and T. Sato, J. Power Sources, 162, 1401 (2006).

4) S. Mitani, S. Lee, K. Saito, Y. Korai, and I. Mochida, Engineering Sciences Reports, Kyushu University, 29, 278 (2007).

5) T. Matsushita, K. Dokko, and K. Kanamura, J. Power Sources, 146, 360 (2005).

6) D. Aurbach and O. Chusid, J. Electrochem. Soc., 140, L155 (1993).

7) K. Kanamura, S. Toriyama, S. Shiraishi, M. Ohashi, and Z. Takehara, J. Elecrtoanal. Chem., 419, 77 (1996).

8) K. Kanamura, H. Takezawa, S. Shiraishi, and Z. Takehara, Denki Kagaku, 66, 272 (1998).

9) K. Kanamura, T. Umegaki, M. Ohashi, S. Toriyama, S. Shiraishi, and Z. Takehara, Electrochim. Acta, 47, 433 (2001).

10) L. J. Hardwick, J. A. Saint, I. T. Licas, M. M. Doeff, and R. Kostecki, J. Electrochem. Soc., 156, A120-A127 (2009).

11) T. Ogata, M. Abe, and K. Matsuki, Electrochim. Acta, 38, 2511 (1993).

12) V. R. Koch, C. Nanjundash, G. B. Appetecchi, and B. Scrasati, J. Electrochem. Soc., 142, L116 (1995).

13) J. A. Vega, J. Zhou, and P. A. Kohl, J. Electrochem. Soc., 156, A253 (2009). 